\title{
LA RELACIÓN CÍVICO-MILITAR EN LOS NUEVOS ESCENARIOS DE SEGURIDAD Y DEFENSA HEMISFÉRICA: SU IMPACTO EN LA RELACIÓN PERUANO-CHILENA
}

\author{
José Miguel Piuzzi Cabrera \\ General de Brigada, Ejército de Chile
}

\section{INTRODUCCIÓN}

El conjunto de cambios que en materia política, social y económica enfrentan, en la actualidad, los países de América del Sur son, bajo todo punto de vista, notables no sólo por su magnitud, sino que además por su incidencia en la vida de las personas. Como pocas veces, el panorama regional nos muestra un subcontinente donde las rivalidades entre los Estados se han reducido significativamente, y en el cual las relaciones de cooperación priman por sobre las de conflicto, lo que se puede observar tanto en la relación vecinal como a nivel de bloques más amplios. Sin embargo, en el ámbito interno, persisten situaciones de gran complejidad, derivadas de problemas económicos, sociales y culturales, en un contexto de grandes transformaciones, muchas de ellas ajenas a la voluntad y decisión de nuestras comunidades.

En lo que se refiere a la función de defensa, el término del bipolarismo mundial trajo consigo la inauguración de una nueva etapa. A partir de entonces vivimos una suerte de transición, desde esquemas de seguridad y defensa dominados por doctrinas que en gran medida se encontraban influidas por un marcado componente ideológico, hacia asuntos formas 0 arquitecturas de seguridad y defensa, donde la agenda responde más bien a temas concretos que a una fórmula 0 compromiso de acción militar conjunta. Pese a ello, no se puede desconocer que la interpretación y el tratamiento de dichos asuntos también se encuentran en transición.

Pero el panorama regional es aún más complejo. Progresivamente, y como ya lo venían anunciando algunos teóricos de las relaciones internacionales, el mundo se ha ido organizando en torno a principios no territoriales en donde también interactúan actores no estatales, tales como las organizaciones intergubernamentales, las no gubernamentales y las corporaciones multinacionales ${ }^{1}$. La actuación de ellos, en el plano global, no sólo ha venido a disputarle al Estado el rol monopólico que ostentó en el pasado, sino que ha contribuido a la disminución de la competencia de éstos, en diversos ámbitos, sobre los cuales ha perdido potestad².

Pearson, S. Frederic; Rochester, J. Martin. 2000. Relaciones Internacionales, situación global en el siglo XXI. Bogotá: Mc Graw Hill, pp. 96-101.

Ohmae, Kenichi. El fin del Estado Nación. 
En este nuevo entorno, civiles y militares se encuentran de pronto envueltos en una serie de procesos altamente dinámicos, que involucran cambios institucionales y estructurales, para avanzar hacia una nueva etapa. El sustento de este cambio se expresa, en todo caso y con bastante claridad, en una evidente primacía de los regímenes democráticos, del Estado de derecho y del respeto de los derechos humanos, así como en una valoración colectiva de los procesos de entendimiento por sobre cualquier situación de conflicto.

A partir de este breve preámbulo deseo iniciar el desarrollo del tema que me ha sido asignado, el cual relaciona, a su vez, tres asuntos de gran relevancia: la seguridad hemisférica, las relaciones cívico militares y las relaciones chileno peruanas.

Para abordarlos, y considerando que en las exposiciones anteriores se han tratado de algún modo estas materias, he estimado conveniente analizarlos desde dos perspectivas que se complementan. La primera será una mirada de conjunto, que pone el acento en los aspectos comunes que presentan la seguridad hemisférica, las relaciones civiles militares y las que se dan entre los Estados. Luego revisaré, en sus aspectos más destacados y separadamente, cada uno de los aspectos en particular, para posteriormente finalizar con una breve reflexión.

\section{UNA MIRADA DE CONJUNTO}

La necesidad de vincular los aspectos sobre los que versa esta exposición bajo una mirada común radica en la complejidad que éstos presentan y, en especial, en algunas razones de orden práctico que paso a enunciar. La primera consiste en que, conceptualmente a lo menos, tienen relación con fenómenos que son comunes, tales como: los de percepción y confianzas, así como la sensibilidad que su tratamiento despierta en algunas ocasiones. La segunda razón es la apreciación-simplemente como hipótesis- que la realidad que se presenta en la región influye de manera similar en los tres aspectos, no sólo por su interrelación y mutua influencia, sino porque, además, los factores que están presentes en la naturaleza primaria en que se sustentan estos tipos de relaciones hacen que el conjunto de cambios y fenómenos que estamos enfrentando en nuestros países y en la región, influyan muy directamente en este tipo de relaciones, es decir, las que dan sustento a un marco de seguridad o de relaciones institucionales o entre Estados.

Para ello me he formulado una pregunta que nos puede servir de eje para orientar la reflexión que se trata de desarrollar en esta exposición. Así, me he planteado la siguiente interrogante: ¿qué tienen en común la seguridad hemisférica, las relaciones cívico militares y las relaciones entre dos Estados como Chile y Perú?

Sin entrar a teorizar, estimo que la respuesta a esta pregunta requiere la presencia de ciertas condiciones mínimas, como son:

a. Existencia de un sustento institucional básico que establezca las formas de relación y contribuya a la mutua confianza. A mi juicio, éste no es otro que la presencia de regímenes políticos democráticos con un alto nivel de legitimidad y una estricta adhesión a los principios que informan el Estado de derecho (componente político institucional).

b. Voluntad o disposición asociativa o de integración en términos sociales. Esto es, primacía de sentimientos y actitudes de unidad y de convivencia por sobre los de conflicto y de segregación (componente social). 
c. Aceptación, práctica y efectiva de las normas comunes, de las "reglas del juego", individual y colectivamente (componente ético funcional).

d. Sustentabilidad operativa, que apunta a la existencia de la posibilidad cierta de articular propuestas, lograr apoyos, y transformarlos en políticas públicas que contengan los intereses de los distintos sectores involucrados en el seno de los Estados, o de los sectores participantes (componente operativo).

Establecidas las condiciones, podríamos afirmar que, en términos generales, aquí se encuentra el bloque estructural básico para cualquier relación formal que asume un proyecto común, desde los ámbitos más pequeños, como pueden ser las relaciones entre grupos sociales, hasta las relaciones entre países, 0 ámbitos mayores de cooperación o asociación.

Pero ahora surge una segunda cuestión: ¿cuál es la realidad regional, o qué caracteriza al entorno sudamericano en lo que afecta al asunto que estamos tratando?

a. La heterogeneidad de nuestro continente, caracterizado por la presencia de realidades disímiles que en importante medida se ven profundizadas por el impacto del proceso de globalización.

b. Una gran variedad de percepciones respecto de la realidad, y cierta confusión e incertidumbre en las personas, en los actores políticos, sociales y económicos, y también, en el ámbito de las instituciones. En un reciente Congreso lberoamericano, en Madrid, uno de los expositores decía que las instituciones armadas están viviendo una suerte de "estrés", dados los cambios en la función de defensa y los niveles tecnológicos, sin considerar la situación interna de cada país.

c. Hay en la región una baja valoración de la democracia, aun cuando no puede derivarse de ello un apoyo o preferencia de regímenes autoritarios u otros. Impresionan en este sentido los informes y el seguimiento de Latín barómetro, en particular, por la baja que ha sufrido en los últimos años el apoyo a la democracia.

d. Existe, además, una escasa integración social y de grupos, lo que también dificulta el articular propuestas y canalizar demandas de la sociedad. Pero, más importante que ello, facilita los particularismos y las expresiones de autosegregación, que muchas veces se ven influidas por la acción de organizaciones de carácter transnacional.

e. Por último, se aprecia un creciente valor de la diversidad, unida a la percepción de la conveniencia de asumir formas de integración o cooperación que provean beneficios mutuos.

Si analizamos cada uno de estos aspectos, y los relacionamos con las condiciones mínimas que, desde una perspectiva común, requieren la seguridad hemisférica, las relaciones cívico militares y las relaciones entre Estados, podemos concluir, de manera preliminar, que el panorama general no presenta grandes ventajas. Por tanto, el entorno 0 ambiente donde se plasman los esfuerzos y la voluntad de avanzar en estos aspectos no es del todo favorable, y excede con creces la acción de los actores locales. Por lo mismo, la necesidad de ir articulando esfuerzos conscientes de las limitaciones existentes debe ser parte de una acción permanente.

De ahí que, poniendo en perspectiva y alineando las condiciones con la realidad y sus características, podemos valorar, en mayor medida, los avances que en los últimos años se han producido en materia de seguridad hemisférica, de relaciones cívico militares y, muy particularmente, en las relaciones peruano chilenas. 


\section{RELACIONES CÍVICO MILITARES EN LOS NUEVOS ESCENARIOS DE SEGURIDAD Y DEFENSA}

Desde antes de la reunión de Ministros de Defensa en Williamsburg, en 1995, y hasta la realizada en Santiago la semana recién pasada (V), han existido dudas y cuestionamientos sobre el modelo de seguridad colectivo delineado por el Tratado Interamericano de Asistencia Recíproca en 1947. Recordemos que la firma del TIAR se entendía como un acuerdo de seguridad hemisférica "destinado a prevenir y reprimir las amenazas y los actos de agresión contra cualquiera de los países de América"3,... "sustentado por los ideales democráticos y la voluntad de permanente cooperación"4, lo que a su vez permitía afrontar eventuales amenazas en un esquema de guerra fría, y en este caso en una zona de influencia de los Estados Unidos 5 .

No obstante, el expreso compromiso asumido de "ayudar a hacer frente al ataque, en ejercicio del derecho inmanente de legítima defensa individual o colectiva...", expresado en el Tratado, como la posterior creación de la OEA, no tuvo un mayor impacto en las situaciones estratégicas que afectaron a los países miembros, ni en la concepción de las amenazas que enfrentaba cada Estado en particular.

A diferencia de Europa, que contó con un mayor grado de institucionalización en materias de seguridad y defensa, los países de América tuvieron una visión pragmática. Derivada del enorme desequilibrio en relación con los de Estados Unidos, prevaleció una especie de subordinación a un diseño mayor, asumiendo que la seguridad hemisférica se consolidaría con el aporte de todos los países en una fuerza común, cuando ella fuese requerida por el TIAR o mediante la OEA, pero siempre en un esquema donde la potencia eje, los Estados Unidos, lideraba y hacía el esfuerzo principal.

Ello provocó que en varias ocasiones se pusiese en duda la efectividad de dicho Tratado, ya que, como "instrumento" definitorio de un régimen internacional, los mecanismos de su convocatoria y uso se restringieron al criterio emanado de Washington. Progresivamente, el sistema de seguridad colectiva fue evidenciando contradicciones, y, más que representar un sistema interamericano de seguridad de real operatividad, quedó circunscrito a un ámbito más bien formal, que no obstante era necesario mantener. Lo cierto es que la fuerza de los hechos se imponía sobre las intenciones, los intereses y las expectativas, a veces divergentes, de los Estados Unidos y América Latina, lo que "derivó pronto en un desencanto y frustración para América Latina. Nuestra región confiaba en que el sistema de seguridad colectivo implicaría un esquema de cooperación similar al Plan Marshall y un compromiso de EE.UU. con el principio de no intervención. Sólo hubo desilusión en ambos aspectos"'.

Tratado Interamericano de Asistencia Recíproca, 1947, considerando $1^{\circ}$.

$4 \quad$ También dentro de los "considerandos" se afirma "que la obligación de ayuda mutua y de común defensa de las Repúblicas Americanas se halla esencialmente ligada a sus ideales democráticos y a su voluntad de permanente cooperación" TIAR, 1947, considerando $6^{\circ}$.

5 Entre otros testimonios, el diario El Mercurio del 3 de septiembre de 1947 expresa en su editorial que "se han reafirmado y vigorizado los conceptos básicos de solidaridad y cooperación interamericana que fueron enunciados en el Acta de Chapultepec. Se ha logrado mejorar los procedimientos interamericanos para resolver por medios pacíficos las controversias, puntualizándose las obligaciones de ayuda mutua y defensa común", y agrega, "se ha condenado formalmente la guerra y de no recurrir a la amenaza 0 al uso de la fuerza, de acuerdo a las disposiciones de las Naciones Unidas".

6 Exposición del Subsecretario de RR.EE. don Heraldo Muñoz, en Revista XXIV Conferencia de Ejército Americanos, Santiago de Chile, diciembre de 2001. 
En la actualidad se ha ido optando por una arquitectura flexible que recoge la realidad de los acuerdos bilaterales y subregionales en materia de seguridad y defensa. Así, el panorama actual se está estructurado de modo diferente, como un sistema de relaciones múltiples en forma de red o telaraña, donde existe una primacía de acuerdos políticos que son esenciales y básicos para estructurar un conjunto de relaciones económicas, comerciales, militares, diplomáticas, de seguridad, culturales, etc., lo que produce una convergencia de intereses también flexible y dinámica entre los distintos actores ${ }^{7}$.

Este nuevo escenario obliga al Estado Nación a forjarse nuevos roles y a no ser pasivo, ajustando y equilibrando las fuerzas divergentes de la globalización y la fragmentación, y asumiendo las nuevas amenazas de un carácter fuertemente transnacional.

En este nuevo escenario, la seguridad hemisférica se ha transformado en un elemento coayudante del desarrollo y de la estabilidad democrática. Así, se han incorporado criterios y acciones que obligan a una participación más activa de los ministerios de Defensa y de Relaciones Exteriores, lo que implica una relación cívico militar más propia de una etapa de normalización, donde el poder político va definiendo la agenda de seguridad y defensa.

En estos últimos años se ha hecho evidente, además, un compromiso más explícito de los actores sociales y políticos con relación a la defensa, que no existía hace algunas décadas. En esta línea, la reciente reunión de Ministros de Defensa, celebrada en Santiago de Chile, destacó, entre otros aspectos, el incremento en el interés de civiles por conocer y participar en materias de seguridad y defensa.

Por lo demás, las características de las nuevas amenazas que, como se ha expresado, a veces superan la consideración de ciertos criterios internos y externos, y en términos de asuntos de prevención -dependiendo de su magnitud- tienden a confundir los niveles políticos y estratégicos, han hecho surgir una relación civil militar más integradora, y una necesaria complementación en los criterios para la toma de decisiones.

En efecto, las nuevas amenazas responden a problemas transversales o transnacionales como el narcotráfico, el tráfico ilícito de armas, el crimen organizado, las migraciones sin control, el terrorismo, la degradación del medio ambiente, la corrupción, la ingobernabilidad y los estallidos sociales, entre otros. Estas amenazas eventualmente pueden llegar a ser más desestabilizadoras que un conflicto clásico, pasando al terreno de la defensa y seguridad cuando superan las dimensiones de un problema policial. De ser así, ellas exigen prevenirlas de manera integral y multidimensional, para alcanzar soluciones coordinadas de acuerdo con los problemas comunes que puedan presentarse, sin perjuicio del respeto a la diversidad de respuestas que cada Estado pueda adoptar.

Más allá de las amenazas y de su necesario impacto en el desarrollo de nuestros pueblos, hay que considerar también el compromiso y la actividad que han asumido los Ministerios de Defensa, en particular a partir de Williamsburg, lo que implica desde liderar el tratamiento de temas de la agenda común, hasta el trabajo en asuntos específicos, incorporando a la sociedad civil para hacerla partícipe de las decisiones. Algo similar se presenta con la necesaria participación que les cabe en la

Escenarios Actuales, Centro de Estudios e Investigaciones Militares año 7, № 4, octubre-noviembre de 2002, Ponencia del Director del CESIM General de Brigada José Miguel Piuzzi Cabrera "Relaciones Internacionales y Seguridad Hemisférica: una visión desde la estrategia". 
toma de decisiones a las comisiones parlamentarias de defensa, que intervienen en la discusión de presupuestos y en la fiscalización de las actividades en que intervienen las Fuerzas Armadas.

En todo caso, en lo que a relaciones cívico militares se refiere, estamos en presencia de un escenario altamente flexible, dependiendo de la situación particular de cada uno de los países. Sin embargo, los avances de los últimos años han sido significativos, lo que se destaca aún más a través de la institucionalización de las reuniones de Ministros de Defensa, donde es la autoridad política quien asume el liderato de los asuntos de seguridad y defensa, derivándose desafíos y tareas que deben ser asumidas por la sociedad toda.

\section{IMPACTO EN LAS RELACIONES CHILENO PERUANAS}

Los cambios evidenciados en los últimos años han contribuido a un posicionamiento de los ministerios de Defensa de la agenda de seguridad y defensa en el continente, y particularmente en nuestros países. Ello ha dado origen a un trabajo que facilita y contribuye a una normalización de las relaciones cívico militares, dentro de la realidad propia y singular de cada país, y en el marco genérico en que se desenvuelven en el panorama sudamericano, con las limitaciones que señalamos en el análisis de conjunto de la primera parte de esta exposición.

Lo anterior contribuye a superar antiguas concepciones en virtud de las cuales se entendía que los temas de seguridad y defensa, asociados por lo demás a los conflictos propios del esquema de la Guerra Fría, sólo debían ser abordados por los institutos armados, o donde más que una integración civil militar acorde a la necesidad de los desafíos actuales, lo central era lograr una supremacía civil como expresión de un control de los institutos armados.

La superación de esta concepción lleva, necesariamente, a la reformulación de las institucionalidades de nuestros respectivos países, a fin de dar cabida a los siguientes aspectos que de algún modo están presentes en nuestra agenda bilateral en esta materia:

- El incremento y fortalecimiento de las reuniones bilaterales de expertos en materias de interés común.

- La adopción de acuerdos explícitos para aunar esfuerzos en el enfrentamiento de las nuevas amenazas, como del intercambio de información indispensable para fortalecer la estabilidad de nuestras instituciones democráticas.

- El desarrollo de un trabajo académico orientado por prioridades similares que constituya una base teórica adecuada para afrontar las incertidumbres que el proceso de globalización ha generado.

- La creciente explicitación de las políticas de defensa para contribuir a la necesaria transparencia en un mundo caracterizado por el profundo cambio en el tipo y en la naturaleza de los conflictos, así como en los consecuentes desafíos que deben abordar nuestras Fuerzas Armadas. Oportuno resulta, en este sentido, acoger la recomendación de la última reunión de Ministros de Defensa de las Américas en cuanto a considerar, para estos efectos, los "Lineamientos sobre la elaboración de documentos sobre políticas y doctrinas nacionales de defensa", confeccionado por la Comisión de Seguridad Hemisférica de la OEA.

- El fortalecimiento de las medidas de confianza mutua en un esquema subregional caracterizado por la primacía de la cooperación. En esta misma línea cobra relevancia el desarrollo de 
esfuerzos sostenidos en orden a la homologación de los gastos de defensa. En este sentido, nuestro país ya cuenta con una fructífera experiencia desarrollada con Argentina.

En ese contexto, Chile aspira a la institucionalización bilateral de un marco de relaciones que haga posible, entre otros aspectos, incrementar la confianza, transparentar los gastos y las políticas de defensa, y construir un ambiente de intercambio metódico y mutuamente beneficioso.

Tales esfuerzos han quedado de manifiesto con la publicación del Libro de la Defensa Nacional de 1997, la elaboración del Libro de la Defensa de 2002, a ser difundido pronto, y en la declaración conjunta de los Presidentes Ricardo Lagos y Alejandro Toledo en Santiago en el mes de julio de 2001.

Dicha declaración constituyó un hito relevante para el desarrollo de nuestras relaciones. Así, en septiembre de 2001 se firma en Lima el primer memorándum de entendimiento destinado a formalizarlas y fortalecerlas en los ámbitos de la cooperación, y se crea el Comité Permanente de Consulta y de Coordinación Política, compuesto por los Ministros de Defensa y Relaciones Exteriores de ambos países (2+2).

El establecimiento de este Comité se inscribe en la convergencia de las voluntades políticas de los dos gobiernos y representa el inicio de una nueva etapa, cualitativamente diferente, en las relaciones para estrechar vínculos en el área de la cooperación en seguridad y defensa, que se fundamenta en el reconocimiento y respeto de los principios y disposiciones de la Carta de las Naciones Unidas y el derecho internacional, en los valores de la democracia y en el propósito de alcanzar mayores niveles de desarrollo.

Se acordóA $\triangle A \approx$ bién en Lima la creación del Comité de Seguridad y Defensa (COSEDE), el cual comenzó a funcionar en octubre de 2001 en la ciudad de Santiago, con la participación de representantes de alto nivel de los ministerios de Defensa y Relaciones Exteriores, y de las fuerzas armadas de ambos países. Simultáneamente se llevó a cabo -en Punta Arenas- la XVI Ronda de Conversaciones entre los Altos Mandos de dichas fuerzas.

Este Comité de Seguridad y Defensa tiene por finalidad evaluar y profundizar las medidas de confianza mutua, desarrollar labores de coordinación y cooperación, y considerar un proceso de consultas con miras a avanzar en la limitación y control de armas convencionales, que someterá a consideración del Comité Permanente ya señalado $(2+2)$.

La segunda reunión de este Comité se desarrolló en Lima, en marzo de 2002, y en agosto de este mismo año los Presidentes de ambos países firmaron en Santiago una declaración conjunta para iniciar los trabajos destinados a proponer una metodología estandarizada común para medir los gastos en defensa.

Otros hitos importantes en las relaciones entre ambos países se relacionan con las Rondas de Conversaciones que han sostenido los Altos Mandos de las FF.AA. desde el año 1986. La última reunión (XVII) se efectuó durante la primera semana de noviembre de este año en Lima, y en ella se analizaron asuntos relativos a ejercicios combinados y conjuntos ante catástrofes (Tsunami), operaciones de paz e incremento de la confianza mutua, y otros referidos a seguridad integral.

Junto con ello, resulta oportuno considerar que los ejércitos de ambos países forman parte de la Conferencia de Ejército Americanos, cuya última versión (XXIV) se realizó en Santiago, en noviembre de 2001, y está previsto que la próxima se lleve a cabo en Canadá durante el año 2003. 


\section{REFLEXION FINAL}

Señoras y señores, distinguidas autoridades:

Es evidente que enfrentamos un mundo en que las certezas tienden a desdibujarse frente a lo vertiginoso de los cambios que se están produciendo. Pero también es cierto que los cambios en el panorama mundial y regional, en particular la necesidad de un trabajo integrado para enfrentar asuntos complejos, además del surgimiento de nuevas amenazas, han fortalecido nuestros intereses comunes.

Podemos, en consecuencia, plantearnos tareas comunes y diseñar esquemas que propendan a ello. La seguridad hemisférica se justifica y puede ser ampliamente asumida en tanto América es sujeto de intereses y de amenazas comunes, para cuya solución la integración de lo político militar es fundamental. Adicionalmente, hay que tener en cuenta, necesariamente, que algunas de estas nuevas amenazas crean un clima de conflicto en el seno de cada sociedad, pudiendo traspasar fronteras, afectando el orden político y poniendo en peligro la paz.

Para evitar un desenlace como éste, es necesario fortalecer el vínculo cívico militar, tal vez en una visión más amplia que la dimensión conceptual institucional que se suele dar.

Si algo está claro en el presente, es que las amenazas del futuro, a diferencia de 1947, cuando surgió el TIAR, no se superan con la sola acción militar de la superpotencia dominante; de hecho cuando se emplea autónomamente ha tenido serios fracasos o no ha logrado sus objetivos (Afganistán 2002). Incluso en esta misma línea es conveniente que los Estados americanos potencien sus vínculos y relaciones, incluidas las cívico militar, para fortalecer su capacidad de influir y cooperar con los Estados Unidos.

La cuestión de fondo consiste en reconocer la existencia de una nueva realidad política y social, en la que conviven intereses y amenazas, que ameritan una respuesta colectiva, porque las repúblicas de América, en un mundo globalizado, son más interdependientes y porque los problemas de inseguridad, y los que en general afectan la paz, tienden a expandirse, ya que sus causas y efectos no reconocen fronteras nacionales.

Muchas gracias.

José Miguel Piuzzi Cabrera es Director del Centro de Estudios e Investigaciones Militares del Ejército de Chile (CESIM), es Oficial de Estado Mayor, Profesor de Historia Militar y Estrategia, egresado del Magíster en Ciencia Política de la Universidad Católica de Chile, Magíster en Ciencias Militares con mención en Planificación y Gestión Estratégica de la Academia de Guerra y Doctor en Sociología de la Universidad Pontificia de Salamanca, España.

(E-mail: pc@entelchile.net) 